\title{
PROSTATECTOMÍA RADICAL EN PACIENTES CON ANTECEDENTES DE RTU PRÓSTATA.
}

\author{
Anna Bujons Tur, María Montlleó González, Xavier Pascual García, Antonio Rosales Bordes, \\ Juan Caparrós Sariol y Humberto Villavicencio Mavrich.
}

Servicio de Urología Fundación Puigvert. Barcelona. España.

\begin{abstract}
Resumen.- OBJETIVO: Nuestro objetivo ha sido realizar una valoración retrospectiva en nuestro centro de las complicaciones quirúrgicas y de morbilidad en pacientes intervenidos de prostatectomía radical retropúbica como tratamiento electivo del cáncer de próstata órgano confinado, con antecedentes de resección transuretral de próstata (RTUp).
\end{abstract}

MÉTODOS: Entre 1980-2004 hemos realizado 59 prostatectomías radicales en pacientes con RTUp previa. Se analiza morbimortalidad de la PRR, resultados funcionales y concordancia entre estadiaje clínico y patológico.

RESULTADOS: El tiempo medio transcurrido entre la RTUp y la prostatectomía radical 16 meses. Edad media en el momento de diagnóstico de adenocarcinoma de próstata 63 años. Estadiaje clínico: 1671 a, 18T1b, 20T1c, 3 T2a, 2 T2b. La media de tiempo quirúrgico de la PRR 180 minutos. Incidencias intraoperatorias: Dificultades técnicas en la disección de la glándula 57\%, preservación del cuello vesical $27 \%$, preservación de bandeletas neurovasculares 3.39\%, ligadura de uréter $1.69 \%$, lesión rectal $1.69 \%$, uretrorragia $1.69 \%$, fístula urinaria $5 \%$ y el $11.8 \%$ requirió transfusión. Como complicaciones postoperatorias: ITU 10.17\%, infección de la herida 10.17\%, hematoma pélvico 5.08\%, TVP $1.69 \%$ y un paciente falleció súbitamente al mes de la cirugía por causa no filiada. Estadío patológico: 49pT2b, 8pT3 y 2pT4. Como complicaciones tardías: Disfunción eréctil $85.7 \%$, estenosis de la anastomosis $10.3 \%$ e incontinencia urinaria total $3 \%$.

CONCLUSIÓN: La PRR en pacientes con RTUp previa es técnicamente más dificultosa con resultados comparables con los pacientes sin cirugía prostática previa.

Palabras clave: Prostatectomía radical. Resección transuretral de próstata. Morbilidad.

Summary.- OBJECTIVES: To perform a retrospective evaluation of surgical complications and morbidity in patients undergoing radical retropubic prostatectomy (RRP) as elective treatment for organ-confined prostate cancer in our center with previous transurethral resection of the prostate (TURP).

METHODS: Between 1980-2004 we performed 59 radical prostatectomies in patients with previous TURP. We analyze the morbidity and mortality of the RRP, its functional outcomes, and the accordance between clinical and pathological stage. 
RESULTS: Mean time between TURP and RRP was 16 months. Mean patient age at the time of diagnosis was 63 years. Clinical stage: 16 Tla, 18T1b,20Tlc, 3T2a,2T2b. Average surgical time was 180 minutes. Intraoperative events: technical difficulties in the dissection of the gland $57 \%$, bladder neck preservation $27 \%$, neuro vascular bundles preservation $3.39 \%$, ureter ligation $1.69 \%$, rectal laceration 1.69 , urethrorrhagia $1.69 \%$, urinary leak 5\%, and blood transfusions $17.8 \%$. Post operative complications: urinary tract infection $10.17 \%$, wound infection $10.17 \%$, pelvic hematoma $5.08 \%$, deep vein thrombosis $1.69 \%$, and one sudden death of unknown cause one month after surgery. Pathologic report: 49pT2b, 8 pT3 and 2pT4. Late complications: erectile dysfunction $85.7 \%$, vesicourethral anastomosis stenosis $10.3 \%$, and complete urinary incontinence $3 \%$.

CONCLUSIONS: Radical retropubic prostatectomy in patients with previous TURP is technically more difficult and has comparable outcomes to RRP patients without previous TURP.

Keywords: Radical prostatectomy. Transurethral resection. Morbidity.

\section{INTRODUCCIÓN}

La prostatectomía radical retropúbica es la intervención quirúrgica de elección en caso de cáncer de próstata órgano confinado (1). La técnica quirúrgica y los resultados han mejorado desde principios de los años 80 , después de los trabajos de Walsh. No obstante, esta cirugía debe de tener como objetivo oncológico, reducir al máximo el índice de positividad de los márgenes de las exéresis quirúrgicas. Todo margen positivo estadísticamente representa una disminución proporcional de supervivencia a largo plazo. Y además conseguir un objetivo funcional con mejora de los resultados de la continencia y de la función eréctil postoperatoria.

El éxito oncológico y funcional de esta cirugía requiere un conocimiento de las estructura anatómicas y una técnica rigurosa de la disección, respetando los límites de exéresis. La prostatectomía radical no está exenta de morbilidad perioperatoria y complicaciones como lesión rectal, estenosis de la unión uretrovesical, incontinencia urinaria y disfunción eréctil. La cirugía previa en la próstata aumenta la dificultad técnica de la prostatectomía radical por la mayor inflamación del tejido periprostático que implica mayor fibrosis que puede condicionar los resultados oncológicos y funcionales.
Presentamos nuestra serie de 59 prostatectomías radicales con antecedentes de RTU próstata analizando morbimortalidad de la PRR, resultados funcionales y concordancia entre el estadío clínico y patológico.

\section{MATERIAL Y MÉTODOS}

Entre 1980-2004 se realizaron en nuestro centro 59 prostatectomías radicales retropúbicas en pacientes diagnosticados de adenocarcinoma de próstata con antecedentes de RTU próstata. La indicación de la RTU prostática había sido en 51 casos desobstructiva en pacientes afectos de hiperplasia benigna de próstata, en 2 biópsica y en 7 desobstructiva-biópsica. Se resecaron $2 \lg (\mathbb{R} 10-81)$ de media de tejido prostático. Como complicaciones de la cirugía endoscópica hubo perforación capsular en 6 pacientes $(10.7 \%)$.

El adenocarcinoma de próstata fue hallado de forma incidental en 34 casos (57.63\%) tras el estudio anatomopatológico de la RTU prostática (16Tla y $18 \mathrm{Tl}$ b); en 25 casos (42.37\%)el estadiaje clínico se había realizado mediante determinación de PSA sérico, cociente PSA libre/PSA total y biopsia prostática transrectal ecodirigida.

La edad media en el momento del diagnóstico de adenocarcinoma de próstata 63 años (R 5370). El valor medio del PSA al diagnóstico $6.3 \mathrm{ng} / \mathrm{ml}$ (R 3-15) y el Gleason más frecuente hallado en la Biopsia transrectal de próstata fue igual o menor a 6 en el $70 \%$ de los casos o igual y mayor a 7 en el $30 \%$. El estadiaje clínico de la neoplasia prostática: $16 \mathrm{Tla}, 18 \mathrm{Tlb}, 20 \mathrm{Tlc}, 3 \mathrm{~T} 2 \mathrm{a}, 2 \mathrm{~T} 2 \mathrm{~b}$. Por lo tanto, la mayoría de los enfermos de la serie presentaban en el momento del diagnóstico un estadío clínico Tl aTlb siendo el Gleason 6 el más habitual de las biopsias prostáticas.

Se analizó el tiempo medio transcurrido entre la RTUp y la prostatectomía radical que había sido de 16 meses (R 5-38).

La técnica quirúrgica utilizada la descrita por Walsh (2). Se realizó linfadenectomía obturatriz en los casos de Gleason 7 bilateral.

El tiempo medio de seguimiento posterior de estos pacientes ha sido de 52 meses (R 4-120).

Mediante base de datos SPSS analizamos morbimortalidad de la PRR, resultados funcionales y concordancia entre estadiaje clínico y patológico. 


\section{RESULTADOS}

Hemos analizado las complicaciones derivadas de la prostatectomía radical intraoperatoriamente, durante el postoperatorio y al año de la cirugía.

\section{Como incidencias intraoperatorias:}

- 34 casos $(57.63 \%)$ con dificultades técnicas durante la disección de la glándula: dificultad en la identificación del ápex prostático y uretra membranosa por presencia de fibrosis, dificultad en la disección apical debido a la incapacidad de reconocer el plano correcto entre la fascia de Denonvilliers y el recto, sólo en 16 casos $(27 \%)$ pudo preservarse el cuello vesical y en $2(3.39 . \%)$ las bandeletas neurovasculares.

- Accidentalmente se ligó el uréter en 1 caso (1.69\%), al disecar la cara posterior del cuello vesical hubo dificultad para reconocer el plano correcto entre la vejiga y las vesículas seminales lesionando los uréteres de forma inadvertida.

- 1 lesión rectal (1.69\%) que se solucionó con sutura primaria en 2 planos de la pared rectal lesionada manteniendo el paciente en dieta absoluta durante 5 días.

- 1 uretrorragia (1.69\%).

El sangrado medio durante el acto quirúrgico $997 \mathrm{ml} \mathrm{(R180-2800)} \mathrm{y} \mathrm{el} 11.8 \%$ requirió transfusión de 1 o más concentrados de hematíes en el momento de la cirugía o en los días posteriores.

El tiempo medio del acto quirúrgico de la PRR fue 180 minutos (R 120-240).

\section{Como complicaciones postoperatorias inmediatas:}

cabe destacar 6 ITU (10.7\%), 3 hematomas pélvicos $(5.08 \%)$ que se solucinaron de forma conservadora, 1 TVP y un paciente falleció súbitamente al mes de la cirugía por causa no filiada. Objetivamos 3 fístulas urinarias en la anastomosis (5\%), resolviéndose el cuadro clínico con actitud conservadora manteniendo la sonda vesical hasta la desaparición de la fuga.

El tiempo medio de ingreso 7.81 días (R 523) y de sondaje vesical 15 días con previa realización de cistografía de relleno para descartar fuga de la anastomosis.

En el estudio anatomopatológico posterior observamos que los estadíos más frecuentes han sido 49 pT2 (83\%), 8 pT3 (14\%) y 2 pT4 (3\%) y Gleason 7 en $70 \%$. Se observa cierto grado de infraestadiaje en el momento del diagnóstico que también refieren la mayoría de series publicadas en la literatura (3-5). (Tabla I) comparativa entre estadíaje clínico y patológico.

Presentaron enfermedad extracapsular el $17 \%$ casos y márgenes positivos en el $19.6 \%$.

En cuanto a complicaciones tardías al año de la cirugía:

- Disfunción eréctil $(86.7 \%)$, como ya hemos comentado anteriormente sólo en 2 casos se pudieron preservar las bandeletas neurovasculares.

- 6 estenosis de la anastomosis uretrovesical (10.3\%) que se corrigieron posteriormente mediante uretrotomía interna tipo Sachse.

- Para la valoración de la continencia, 35 presentaban continencia total $(59 \%), 18$ continencia parcial clasificada en leve $(31 \%)$ con presencia de pérdidas a grandes esfuerzos y sin requerirr uso de PADS, moderada $(7 \%)$ con pérdidas a medianos esfuerzos con uso de 1-2 PADS al día y 6 pacientes con incontinencia total $(3 \%)$.

- Recidiva bioquímica en 10 casos (16.95\%)

\section{DISCUSIÓN}

La prostatectomía radical es el "gold" estándar para el tratamiento del carcinoma de próstata localizado. No obstante, no está exenta de morbilidad perioperatoria y complicaciones como lesión rectal, estenosis de la unión uretrovesical, incontinencia urinaria y disfunción eréctil. Los fines de la prostatectomía radical son la curación de la enfermedad, control urinario y conservación de la función eréctil (6).

TABLA I. TABLA COMPARATIVA ENTRE ESTADIAJE CLÍNICO-PATOLÓGICO.

\begin{tabular}{|lc|l|}
\hline $16 \mathrm{~T} 1 \mathrm{a}$ & $(27 \%)$ & pT2 en $49(83 \%)$ \\
\hline $18 \mathrm{~T} 1 \mathrm{~b}$ & $(30 \%)$ & \\
\hline $20 \mathrm{~T} 1 \mathrm{c}$ & $(34 \%)$ & pT3 en $8(14 \%)$ \\
\hline $3 \mathrm{~T} 2 \mathrm{a}$ & $(5 \%)$ & \\
\hline $2 \mathrm{~T} 2 \mathrm{~b}$ & $(4 \%)$ & pT4 en $2(3 \%)$ \\
\hline Gleason 6 & $(70 \%)$ & Gleason $7(70 \%)$ \\
\hline
\end{tabular}


La cirugía previa en la próstata aumenta la dificultad técnica de la prostatectomía radical (7) por la mayor inflamación del tejido periprostático que implica mayor fibrosis, mayor dificultad en la disección de las vesículas seminales, fascia de Denonvilliers y también para la preservación de bandeletas.

En nuestra serie observamos mayor dificultad en la identificación del ápex prostático y uretra membranosa por la presencia de fibrosis, aumentada en los 2 casos con antecedentes de perforación capsular durante la RTUp. También observamos mayor dificultad en la disección apical debido a la incapacidad para reconocer el plano correcto entre la fascia de Denonvilliers y el recto. Como ya se ha comentado, en nuestro serie tuvimos una lesión rectal $(1.6 \%)$, porcentaje muy similar al resto de series revisadas (8).

Nichols y cols. (9) publicaron que la prostatectomía radical tenía menor dificultad si se realizaba al menos 6 semanas después de la RTUp. Bass y Barret (10) no obstante recomendaban al menos esperar 4 meses. En nuestra serie cabe destacar un tiempo medio de espera de 16 meses (5-38), no hallando resultados estadísticamente significativos en cuanto al tiempo de espera y menor dificultad técnica.

Un 5\% presentó fuga por anastomosis. Ninguno fue reintervenido por este motivo $y$ en todos ellos se adoptó una actitud expectante manteniendo la sonda vesical hasta la resolución espontánea de la misma. El porcentaje de márgenes positivos en nuestra serie $19.6 \%$ es similar a otros grupos de prostatectomías radicales sin RTUp (Maffezzini 21.3\%, Scardino $12.9 \%)(3,7)$.

En cuanto a las complicaciones tardías, la presencia de estenosis uretrovesical en el $10.3 \%$ de nuestra serie puede ser debida a que la resección transuretral de próstata o la reconstrucción habría dado lugar a una mayor cicatrización y a la formación de una estenosis secundaria. La pérdida sanguínea, los antecedentes ya mencionados y la extravasación de orina en el sitio de la anastomosis podrían contribuir al desarrollo de la estenosis. Cualquier factor que interfiera en la aposición de las mucosas aumenta su riesgo (7). En la literatura la frecuencia de estenosis de anastomosis después de la prostatectomía radical $0.5 \%-9 \%$, con una serie que informaba estenosis en el $17 \%$ de 465 casos (Geary y cols.) (11). Como ya hemos comentado estos casos fueron resueltos mediante uretrocistoscopia y uretrotomía interna tipo Sachse asociando en ocasiones resección transuretral del tejido cicatricial, requiriendo en alguna ocasión de nuevo uretrotomía por recidiva de la estenosis.
Otra complicación de la cirugía radical es la disfunción eréctil (DE). En nuestra serie hemos observado un $86.7 \%$ de pacientes afectos de DE al año de la intervención, con posibilidad de preservación de las bandeletas neurovasculares sólo en 2 casos por su dificultad técnica en su disección. Estos pacientes presentaban función eréctil antes de la cirugía, edad $<65$ años y recuperaron la potencia sexual a los 3 meses de la cirugía. La prostatectomía radical anatómica con conservación de los nervios ha mejorado nuestros conocimientos sobre las estructuras implicadas en el control urinario y la función eréctil, así como el impacto que ejerce sobre ambos la manipulación quirúrgica. Los índices de continencia y potencia sexual obtenidos por diversos cirujanos varían ampliamente, no obstante son más los pacientes que siguen siendo sexualmente potentes tras la operación, y que la continencia tiende a recuperarse más rápidamente y más completamente que antes (12).

Catalona y cols. (13) Informaron resultados en un estudio de 1870 pacientes después de 18 meses de la prostatectomía radical: el $68 \%$ de los pacientes mantuvo su potencia si se preservaban ambos heces neurvasculares, en comparación con el $47 \%$ cuando se conservaba un haz neurovascular y casi nula sin su preservación.

La frecuencia de la incontinencia tras una prostatectomía radical ha sido motivo de controversia en los últimos años, debido a la discordancia de las cifras publicadas. La frecuencia de la incontinencia urinaria ha disminuido en los últimos 10 años debido con la mayor probabilidad a los avances de la técnica quiúrgica. Pese a esta mejoría, la incontinencia secundaria a la prostatectomía se ha convertido en una importante cuestión en la práctica urológica, que exige los mayores esfuerzos en cuanto a prevención, diagnóstico y tratamiento.

Debido a que se ha observado que la incontinencia disminuye con el tiempo desde la cirugía, la duración del seguimiento es importante. La mayoría de los investigadores están de acuerdo en que es necesario un periodo de seguimiento postoperatorio de 12 meses antes de decidir sobre el estado de continencia. En la Tabla II se comparan los porcentajes de incontinencia de diferentes series (14-16).

A nuestro criterio, llama la atención la comparación de tasa de incontinencia urinaria tras prostatectomía radical de nuestro centro con un $0.8 \%$ de incontinencia total y un $7 \%$ de incontinencia a grandes esfuerzos con nuestros casos de prostatectomía radical con RTUp analizados con un incremento al $3 \%$ de incontinencia total, $7 \%$ incontinencia urinaria moderada con pérdidas a medianos esfuerzos que 
requieren el uso de 1-2 PADS al día, 31\% de continencia urinaria muy leve, con pérdidas de orina a grandes esfuerzos y sin necesidad de uso de PADS y sólo un $59 \%$ de continencia total. Por lo tanto, hemos encontrado resultados estadísticamente significativos en cuanto a la tasa de incontinencia urinaria de los pacientes operados de prostatectomía radical con antecedentes de RTUp $(p<0.05)$.

Es importante realizar una disección cuidadosa, no hemorrágica del complejo venoso preprostático, ya que permite visualizar previamente el vértice prostático, respetar el esfínter durante la disección de la uretra y conservar el plexo pélvico y sus ramas que aportan la inervación autónoma y somática del esfínter y en estos casos una mayor dificultad de identificación de las estructuras debido a la fibrosis puede condicionar el mantenimiento del mecanismo de la continencia. Además, la conservación del cuello vesical o su reconstrucción con una anastomosis de buena calidad es un elemento que mejora la continencia postquirúrgica, y en nuestra serie sólo se pudo preservar el cuello vesical en 16.

Encontramos, por lo tanto relación estadísticamente significativa entre la preservación de cuello vesical y tasa de continencia y relación entre la RTUp y poca probabilidad de preservación del cuello vesical. También analizamos si existía relación entre el número de gramos resecados $(<30$ y $>40 \mathrm{~g})$ y el mecanismo de continencia y no hallamos diferencias estadísticamente significativas.

La serie de Nichols y cols. (9) presentaron una tasa de incontinencia del $15 \%$ y de incontinencia de esfuerzo del $42 \%$ en 33 pacientes operados de prostatectomía radical con previa cirugía de la próstata. Bass y Barret (10) publicaron una tasa de incontinencia total del $11 \%$, y $39 \%$ con mínima incontinencia de esfuerzo en una serie de 36 pacientes. No obstante, según Steiner y cols. (15) no existían diferencias en cuanto a la continencia en los pacientes con cirugía prostática previa de una serie analizada de 593 prostatectomías radicales, de las cuáles 84 tenían antecedentes de cirugía previa no se observaron diferencias estadísticamente significativas. Al igual que Ramon y cols. (17) que en un grupo de 153 pacientes con antecedentes de RTUp, un $4 \%$ presentó incontinencia urinaria de esfuerzo, mientras que no hubo ningún incontinente.

El mecanismo de la preservación del esfínter uretral distal es el mayor factor de mantenimiento de la continencia en la prostatectomía. Si el esfínter uretral no es respectado durante la RTUp o durante la disección apical del ápex es dificultoso el mantenimiento de la continencia (18).

En nuestra serie tuvimos recidiva bioquímica en un $16.95 \%$ y presencia de márgenes positivos de $19.6 \%$, similar a otras series publicadas $(19,20)$.

\section{CONCLUSIÓN}

La prostatectomía radical es el tratamiento de elección para el cáncer de próstata localizado. A pesar de ello, en los pacientes con RTUp puede comportar una morbilidad, en ocasiones no despreciable para el enfermo y es aconsejable poner en conocimiento del paciente antes de decidir la mejor terapéutica.

TABLA II. TASAS DE INCONTINENCIA URINARIA POST-PROTATECTOMÍA RADICAL.

\begin{tabular}{|l|l|l|l|}
\hline Autor & Número de casos & Incontinencia total & IUE $\%$ \\
\hline Ingel et al. & 692 & 5 & 10 \\
\hline Lindner et al & 105 & 5 & 13 \\
\hline O'Donnell et al. & 34 & 0 & 6 \\
\hline Walsh et al & 119 & 0 & 2,5 \\
\hline Rossignol et al. & 115 & 0 & 4 \\
\hline Pederson et al & 67 & 7 & 21 \\
\hline Narayan et al. & 125 & 2 & 8 \\
\hline Klein & 60 & 2 & 10 \\
\hline Fund.Puigvert & 501 & 0,8 & 7 \\
\hline
\end{tabular}


Técnicamente es más dificultosa por presencia de fibrosis del tejido periprostático, mayor dificultad en la realización de anastomosis vesicouretral a causa de la rigidez del cuello vesical después de la RTUp mayor dificultad para la realización de la anastomosis sin tensión y para la preservación de las bandeletas neurovasculares. También presenta mayor riesgo de sangrado y tiempo quirúrgico.

Las complicaciones tardías como la estenosis del cuello vesical (10.3\%), mayor riesgo de incontinencia total $(3 \%)$, similar tasa de impotencia $(85 \%)$ en los casos de no preservación de los haces neurovascualres, similar riesgo de márgenes positivos $(19.6 \%$ y de recidiva bioquímica (16.95\%).

\section{BIBLIOGRAFIA y LECTURAS RECOMENDADAS (*lectura de interés $y^{* *}$ lectura fundamental)}

1. WALSH, P.: "Anatomic radical prostatectomy". In. Walsh P, Retik A, Vaughan E, Wein A eds. Campbell's urology. Philadelhia: Wb Saunders, 2565-2588, 1998.

2. WALSH, P.C.; LEPOR, H.; EGGLESTON, J.C.: "Radical prostatectomy with preservation of sexual function: anatomical and pathological considerations". Prostate, 4: 473, 1983.

*3. MAFFEZZINI, M.; SEVESO, M.; TAVERNA, G. y cols.: "Evaluation of complications and results in a contemporany series of 300 consecutive radical retropubic prostatectomies with the anastomotic approach at a single institution". Urology, 61: 982, 2003.

4. RIOJA, L.A.; LIEDANA, J.M.; RONCALES, A. y cols.: "Análisis de una serie de prostatectomías radicales". Actas Urol. Esp., 21: 808, 1997.

5. PORTILLO, J.; RADO, M.; GUTIÉRREZ, J.L. y cols.: "Complicaciones de una serie consecutiva de 133 casos de prostatectomías radicales". Actas Urol. Esp., 25: 559, 2001.

6. American Urological Association Education and Research Guidelines.

7. BARRÉ, C.; CHAVEAU, P.: "Prostatectomía radical retropúbica". Encyclopedie Médico-Chirurgicale-E-41-295, 2003.

**8. LEPOR, H.; NIEDER, A.; FERNANDINO, M.: "Intraoperative and postoperative complications of radical retropubic prostatectomy a consecutive series of 1000 cases". J. Urol., 166: 1729, 2001.

9. NICHOLS, R.T.; BARRY, J.M.; HODGES, C.V.: "The morbidity of radical prostatectomy for multifocal stage I prostatic adenocarcinoma". J. Urol., 117: 83, 1977.
10. BASS, R.B. Jr.; BARRETT, D.M.: "Radical retropubic prostatectomy after transurethral prostatic resection". J. Urol., 124: 495, 1980.

11. GEARY, E.S.; DENDINGER, T.E.; FREIHA, F.S. y cols.: "Incontinence and vesical neck strictures following radical retropubic prostatectomy". Urology, 45: 1000, 1995.

12. MANSFIELD.: "Seminars in Urologic Oncology". 14: 174, 1996.

13. CATALONA, y cols.: Urol. Int., 72: 17, 2000.

14. MARTÍNEZ-CÁCERES, P. y cols.: "Incontinencia post-prostatectomía radical". Actas Fund. Puigvert, 20: 36, 2001.

15. STEINER, M.S.; MORTO, R.A.; WALSH, P.C.: "Impact of anastomotical radical prostatectomy on urinary continence". J. Urol., 145: 512, 1991.

16. AGUILÓ, F.; SUÁREZ NOVO, J.F.; PLANES MORÍN, J.: "Prostatectomía radical. Revisión de nuestra serie en el periodo 1997-2003". Actas Urol. Esp., 29: 542, 2005.

17. RAMON, J.; ROSSIGNOL, G.; LEANDRI, P. y cols.: "Morbidity of radical retropubic prostatectomy following previous prostatic resection". J. Surg. Oncol., 55: 14, 1994.

18. EPSTEIN, J.I.: "Evaluation of radical prostatectomy capsular margins of resection: The significance of margins designated as negative, closely approaching, and positive". Am. J. Surg. Pathol., 14: 626, 1990.

19. BANDHAUER, K.; SENN, E.: "Radical retropubic prostatectomy after transurethral prostatic resection". Eur. Urol., 15: 180, 1988.

20. ELDER, J.S.; GIBBONS, R.P.; CORREA, R.J. y cols.: "Morbidity after radical perineal prostatectomy following transurethral prostatic resection". J. Urol., 124: 495, 1980.

\section{COMENTARIO EDITORIAL}

En nuestra experiencia, los pacientes tratados con prostatectomía radical clásica, tras haber sufrido una RTUP, tienen más tasa de incontinencia que los pacientes vírgenes. Todavía no tenemos suficientes casos de prostatectomía laparoscópica con RTUP previa para saber si también en estos ocurre lo mismo. Los autores llegan a la misma conclusión en el texto pero en el resumen no lo dejan suficientemente claro en mi opinión. Creo que es muy importante advertir a estos pacientes que tienen un riesgo mayor de incontinencia, que a mi juicio, es la peor complicación de esta cirugía.

E. Pérez-Castro Editor 